\title{
Serum Antithyroglobulin Antibody Levels Are Associated with Diabetic Retinopathy among Euthyroid Type 2 Diabetes Patients: A Hospital-Based, Retrospective Study
}

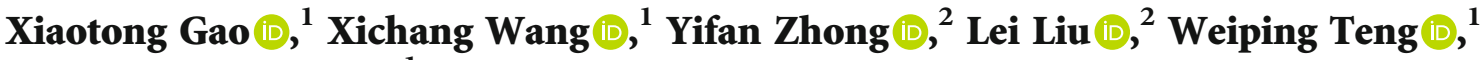 \\ and Zhongyan Shan ${ }^{1}$ \\ ${ }^{1}$ Department of Endocrinology and Metabolism and the Institute of Endocrinology, The NHC Key Laboratory of Diagnosis and \\ Treatment of Thyroid Diseases, The First Hospital of China Medical University, Shenyang 110001, China \\ ${ }^{2}$ Department of Ophthalmology, The First Hospital of China Medical University, Shenyang 110001, China
}

Correspondence should be addressed to Lei Liu; liuleijiao@163.com and Zhongyan Shan; cmushanzhongyan@163.com

Received 29 July 2021; Revised 10 December 2021; Accepted 13 December 2021; Published 6 January 2022

Academic Editor: Eusebio Chiefari

Copyright ( 2022 Xiaotong Gao et al. This is an open access article distributed under the Creative Commons Attribution License, which permits unrestricted use, distribution, and reproduction in any medium, provided the original work is properly cited.

\begin{abstract}
Background. Previous studies have revealed that the variation of thyroid indicators may be associated with the risk of diabetic retinopathy (DR) among euthyroid type 2 diabetes (T2D) patients. But the specific conclusions are currently inconsistent. Methods. This is a hospital-based retrospective survey. We recruited 1,145 euthyroid T2D patients and checked the thyroid function and fundus photographs. The modified Airlie House classification system was used to categorize the stages of DR. The association between thyroid indicators and different stages of DR was analyzed. Results. We divided free triiodothyronine (FT3) into tertiles and found that the prevalence of mild nonproliferative DR (NPDR) was significantly higher in T2, compared with T1 (32.0\% vs. $25.2 \%, p<0.05)$. When FT3 was within the level of T2, FT3 could be an independent risk factor for mild NPDR (OR 1.426, 95\% CI $(1.031,1.971), p<0.05)$. In addition, the prevalence of severe NPDR and proliferative DR (PDR) was significantly higher in thyroglobulin antibody (TgAb) positive group $(8.8 \%$ vs. $4.1 \%, p<0.05)$ and vice versa $(33.3 \%$ vs. $18.4 \%, p<0.05)$. TgAb positivity was also an independent risk factor for severe NPDR and PDR (OR 2.212, 95\% CI (1.244, 3.934), $p<0.05)$. Conclusions. We hardly observed a significant change in DR risk with the elevation or reduction of serum TSH or thyroid hormone within the reference interval. Although the slightly elevated FT3 may be associated to mild NPDR, the extensibility of this result remains to be seen. For T2D patients with euthyroid function, there may be a significant correlation between serum TgAb positivity and severe NPDR and PDR.
\end{abstract}

\section{Introduction}

Thyroid dysfunction and diabetes are the two most common endocrine diseases in clinical practice. A recent national cross-sectional study has revealed that in mainland China the weighted prevalence of thyroid dysfunction and diabetes have reached up to $15.33 \%$ and $12.88 \%$, respectively $[1,2]$, and a huge amount of evidence have demonstrated the potential association between them [3]. Type 2 diabetes (T2D) is currently the most common type of diabetes, accounting up to $95 \%$ of overall diabetes [4]. Several previous studies have demonstrated a significantly higher preva- lence of thyroid dysfunction among T2D patients and vice versa [5-11]. Subclinical hypothyroidism $(\mathrm{SCH})$ is recognized as the most common type of thyroid dysfunction [1], and there are currently the most studies on $\mathrm{SCH}$ in the relevant investigations on thyroid dysfunction and diabetic complications. Han et al. have meta-analyzed ten studies and found that there was a 2.32 -fold increasing risk for $\mathrm{SCH}$, among patients with T2D [12]. Based on the same meta-analysis, the prevalence risks of diabetic nephropathy $(\mathrm{DN})$, diabetic retinopathy (DR), peripheral arterial disease, and neuropathy were also positively associated with $\mathrm{SCH}$ [12]. In addition, several studies have found that although 


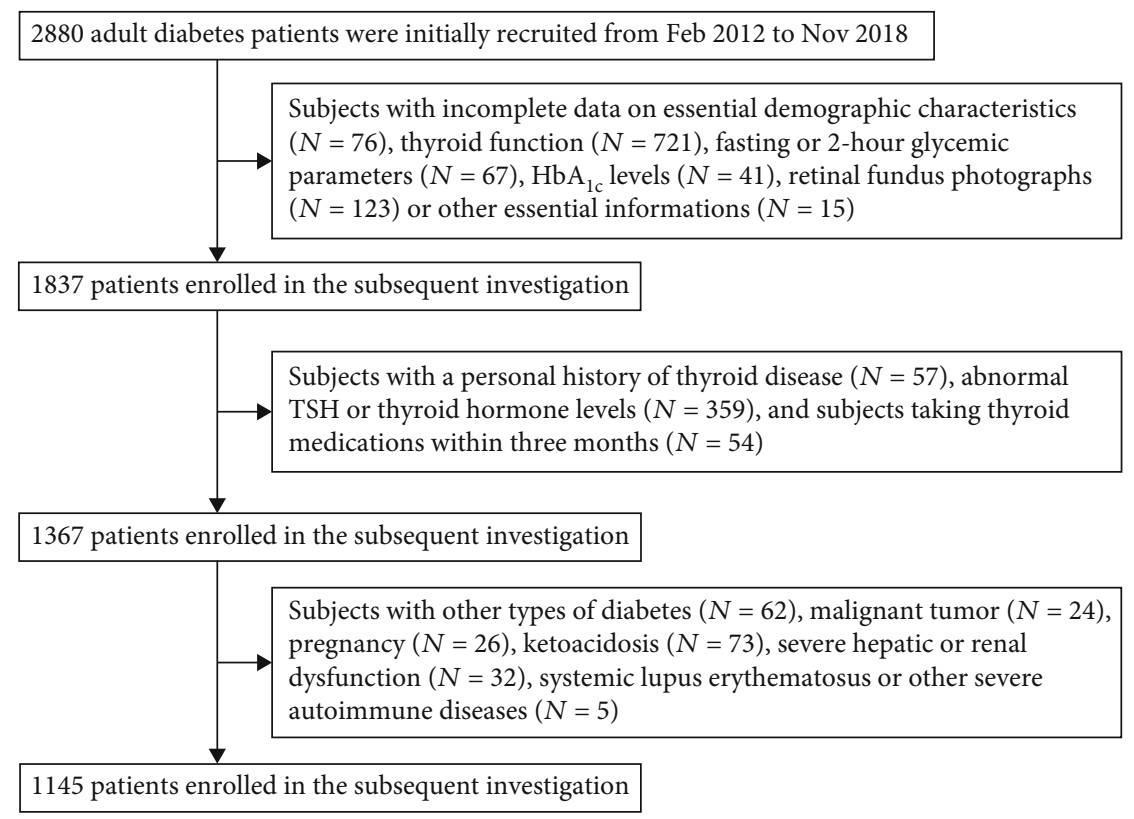

FIGURE 1: The screening process of the participants.

the prevalence of hyperthyroidism in T2D patients is far lower than hypothyroidism, it is generally higher than in the normoglycemic population $[9,13,14]$.

As for the prevalence risk of T2D and its complications among euthyroid subjects, there are currently few but inconsistent conclusions. Since the variation of thyroid function within the normal range may also be associated with poor glycemic control in T2D patients $[15,16]$, there must be a potential association between thyroid indicators and T2D complications in euthyroid subjects. However, the evidence is currently scarce, and there are a few discrepancies in the relevant conclusions [17-19]. Therefore, we need to carry out more studies to analyze whether various thyroid indicators are associated with T2D complications among euthyroid subjects.

As the leading cause of moderate to severe blindness in working aged adults, as well as the most common and specific microvascular complication, DR has received more and more extensive attention in recent years [20, 21]. Optimal control of hyperglycemia, hypertension, and dyslipidemia remains the foundation for reduction of DR development and progression [20]. However, in light of the higher prevalence of thyroid dysfunction among T2D patients, it is necessary to explore the potential impact of thyroid indicators on the prevalence and development of DR. There is currently insufficient evidence in this field, especially for euthyroid subjects. In this hospital-based retrospective survey, we conducted an association analysis for euthyroid T2D patients, in order to supplement and innovate the relevant previous conclusions.

\section{Materials and Methods}

2.1. Study Participants. The present study was approved by the Medical Ethics Committee of the First Affiliated Hospital of China Medical University. The protocol of this hospital- based retrospective study has been previously described in another article [22]. Briefly speaking, we recruited 2,880 diabetic patients aged $\geq 18$ years between February 2012 and November 2018. The diagnostic criteria for diabetes were derived from the 2019 American Diabetes Association (ADA) guidelines [4]. If the patient met one of the four following items, diabetes could be diagnosed: fasting plasma glucose $(\mathrm{FPG}) \geq 7.0 \mathrm{mmol} / \mathrm{L}$, glycosylated hemoglobin $\left(\mathrm{HbA}_{1 \mathrm{c}}\right) \geq 6.5 \%$, 2-hour plasma glucose $(2 \mathrm{~h}-\mathrm{PG}) \geq$ $11.1 \mathrm{mmol} / \mathrm{L}$, with classic symptoms plus random plasma glucose $\geq 11.1 \mathrm{mmol} / \mathrm{L}$. In addition, patients who reported a personal history of diabetes were also recognized as diabetes patients. All patients signed an informed consent form and then completed the questionnaire survey. Information about demographics, personal history of diseases, and medications was all covered by the questionnaire.

The screening process of the study population is shown in Figure 1. We first excluded patients with incomplete essential information, and then patients with a personal history of thyroid disease, abnormal TSH or thyroid hormone levels (FT3 or FT4), and those who had taken thyroid drugs within 3 months were also excluded. To avoid the impact of nonthyroid disease syndrome (NTIS) or pregnancy on thyroid function, patients with malignancy, ketoacidosis, severe hepatic or renal failure, other forms of diabetes, or severe autoimmune diseases, as well as pregnant women were also excluded. Finally, 1,145 euthyroid T2D patients were involved in the final analysis.

2.2. Clinical and Laboratory Measurement. As previously described [22], weight and height were measured by trained nurses, and body mass index (BMI, $\mathrm{kg} / \mathrm{m}^{2}$ ) was calculated as weight divided by the square of the height. Systolic BP (SBP), diastolic BP (DBP), and heart rate (HR) were measured three times with standard mercury sphygmomanometers, and the average was regarded as the final value. After 
an overnight fasting ( $\geq 8$ hours), venous blood samples were taken and immediately transferred to the Endocrinology Laboratory of China Medical University for further measurements.

The following fasting biochemical parameters could be queried in the Hospital Information System (HIS), which mainly include high-density lipoprotein (HDL, mmol/L), low-density lipoprotein (LDL, $\mathrm{mmol} / \mathrm{L}$ ), triglyceride (TG, $\mathrm{mmol} / \mathrm{L})$, total cholesterol (TC, $\mathrm{mmol} / \mathrm{L})$, FPG $(\mathrm{mmol} / \mathrm{L})$, fasting insulin (FINS, $\mathrm{mU} / \mathrm{L}$ ), fasting $\mathrm{C}$ peptide (FCP, $\mathrm{pmol} / \mathrm{L})$, and $\mathrm{HbA}_{1 \mathrm{c}}(\%)$. In addition, all the participants went through a 2 -hour $75 \mathrm{~g}$ oral glucose tolerance test $(2 \mathrm{~h}$ OGTT). We also recorded the results of $2 \mathrm{~h}-\mathrm{PG}, 2 \mathrm{~h}-\mathrm{INS}$, and $2 \mathrm{~h}-\mathrm{CP}$ after taking the glucose. The specific testing instruments of the above indicators were the same as the previous study [22]. What is more, serum TSH, FT3, FT4, thyroid peroxidase antibody (TPOAb), and TgAb were tested with supersensitive chemiluminescence immunoassay (ARCHITECT system i2000SR, Abbott, Chicago, US). The five thyroid parameters of each patient were also queried by trained nurses from the HIS.

2.3. Diagnostic Criteria of Thyroid Dysfunction and Dyslipidemia. As defined by the manufacturer, the reference interval of serum TSH, FT3, FT4, TPOAb, and TgAb was $\quad 0.35-4.94 \mathrm{mU} / \mathrm{L}, 2.43-6.01 \mathrm{pmol} / \mathrm{L}, 9.01-19.05 \mathrm{pmol} / \mathrm{L}$, $\leq 5.61 \mathrm{IU} / \mathrm{mL}$, and $\leq 4.11 \mathrm{IU} / \mathrm{mL}$, respectively. Euthyroid was defined as normal TSH plus normal free thyroid hormone (FT3 and FT4) levels, with or without positive TPOAb or TgAb. The diagnostic criteria for dyslipidemia and hyperuricemia were also defined by the kit. If the subject met all the following four items and was not taking lipid-lowering drugs, it could be considered as normal lipidemia; otherwise, dyslipidemia was diagnosed: $\mathrm{HDL} \geq 0.91 \mathrm{mmol} / \mathrm{L}, \mathrm{LDL} \leq$ $3.64 \mathrm{mmol} / \mathrm{L}, \mathrm{TG} \leq 1.7 \mathrm{mmol} / \mathrm{L}$, and $\mathrm{TC} \leq 5.72 \mathrm{mmol} / \mathrm{L}$. The upper limit of the reference value of uric acid is $428 \mu \mathrm{mol} / \mathrm{L}$. If the level of serum uric acid was higher than the above value or the participant was currently taking hypouricemic drugs, then hyperuricemia would be diagnosed.

2.4. Assessment and Stratification of DR. A nonmydriatic fundus camera (CR6-45NM; Canon, Tokyo, Japan) was used to evaluate the presence and severity of DR. Two-field fundus examination focusing on the fovea and optic disk was applied by trained ophthalmologists to evaluate the location and extent of lesions. The specific protocol of fundus examination has been previously introduced [22]. According to the Early Treatment for Diabetic Retinopathy Study (ETDRS), the modified Airlie House classification system was referred to evaluate the severity of DR [23], which grouped DR into nonproliferative DR (NPDR) and proliferative DR (PDR). NPDR was further divided into mild, moderate, and severe NPDR.

2.5. Statistical Analysis. The above data were input into the Statistical Package for the Social Sciences version 25 (SPSS Inc., Chicago, IL, USA). All $p$ values obtained were based on two-tailed tests, with significance level set at 0.05 . In the descriptive analysis, continuous variables were described as means and standard deviations (SD), and dichotomous variables were described as numbers and corresponding percentages. Single-sample $t$-test and chi-square test were, respectively, used to compare the differences in continuous and dichotomous variables, both between the two groups with or without DR, as well as between the groups with different levels of thyroid function. Multivariate logistic regression was used to analyze the influence of different thyroid parameters on the prevalence DR.

In order to explore whether different concentrations of serum thyroid indicators are related to the risk of different stages of DR, we divided the thyroid indicators, i.e., TSH, FT3, and FT4, into three tertiles (T1-T3) according to the principle of equalizing the number of people in each group. T2 and T3 were assessed to explore the variation of DR risk with the elevation of the three indicators, compared with T1. In addition, subjects were also divided into two groups according to the values of TPOAb and $\operatorname{TgAb}$ (i.e., positive group and negative group).

\section{Results}

3.1. General Characteristics of the Patients. As shown in Table 1, the patients were grouped into two groups by the present of DR. The present study included 719 T2D patients without DR and $426 \mathrm{DR}$ patients. Anthropometric, demographic, and biochemical information were listed and compared between patients with and without DR.

We found that T2D patients with DR were significantly older than those without DR $(p<0.01)$. Compared with T2D patients without DR, DR patients had a significantly lower BMI $(p=0.04)$. What is more, the HR and SBP values were both significantly higher in the DR group $(p<0.01)$. However, the difference in the prevalence of hypertension between DR and non-DR patients was not significant.

As for the comparison of serum biochemical indicators (Table 1), we found that the level of plasma $\mathrm{HbA}_{1 \mathrm{c}}$ was significantly higher in the DR group $(p=0.04)$. However, the remaining plasma glycemic indicators did not show significancy between the two groups, whether it was a fasting or a 2-hour indicator. Moreover, regarding the difference in the homeostasis model assessment of insulin resistance (HOMA-IR), DR patients had a slightly but insignificantly higher degree of insulin resistance.

3.2. The Changing Patterns of Thyroid Indicators with Different Stages of DR. Due to the extremely low prevalence of PDR $(17 / 1,145)$, to ensure the reliability of the results of the investigation, patients with severe NPDR or PDR in this study were combined into one group. According to the presentation and severity of retinopathy, the subjects were divided into four groups, and the serum thyroid index levels between the groups were compared.

As shown in Table 2, except for the positive rate of serum $\operatorname{TgAb}$, there was no statistical difference in the remaining four thyroid indicators among subjects in different groups. We found that the positive rate of serum $\mathrm{TgAb}$ had a significantly increasing trend ( $p$ for trend $<0.05$ ), with the continuous aggravation of ocular lesions. Moreover, 
TABLE 1: General characteristics of participants stratified by the presence of DR.

\begin{tabular}{|c|c|c|c|c|}
\hline Number $(N, \%)$ & $\begin{array}{l}\text { Total } \\
1,145\end{array}$ & $\begin{array}{c}\text { Non-DR } \\
719\end{array}$ & $\begin{array}{l}\mathrm{DR} \\
426 \\
\end{array}$ & $p$ value \\
\hline Sex (female, \%) & $494(43.1 \%)$ & $305(42.4 \%)$ & $189(44.4 \%)$ & 0.52 \\
\hline Age (years) & $56.30 \pm 13.32$ & $55.45 \pm 14.11$ & $57.73 \pm 11.74$ & $<0.01$ \\
\hline T2D duration (>10 years, \%) & $726(63.4 \%)$ & $443(61.6 \%)$ & $283(66.4 \%)$ & 0.10 \\
\hline Smoking $(N, \%)$ & $1,017(88.8 \%)$ & $635(88.3 \%)$ & $382(89.7 \%)$ & 0.48 \\
\hline Drinking $(N, \%)$ & $1004(87.7 \%)$ & $628(87.3 \%)$ & $376(88.3 \%)$ & 0.64 \\
\hline $\operatorname{BMI}\left(\mathrm{kg} / \mathrm{m}^{2}\right)$ & $25.62 \pm 3.78$ & $25.75 \pm 3.94$ & $25.40 \pm 3.50$ & 0.04 \\
\hline HR (per min) & $81.34 \pm 11.19$ & $80.78 \pm 10.82$ & $82.30 \pm 11.74$ & $<0.01$ \\
\hline SBP (mmHg) & $135.15 \pm 20.28$ & $133.77 \pm 19.25$ & $137.49 \pm 21.74$ & $<0.01$ \\
\hline DBP (mmHg) & $82.50 \pm 11.88$ & $82.08 \pm 11.75$ & $83.19 \pm 12.09$ & 0.06 \\
\hline Hypertension $(N, \%)$ & $579(50.6 \%)$ & $348(48.4 \%)$ & $231(54.2 \%)$ & 0.06 \\
\hline Hyperuricemia $(N, \%)$ & $113(9.9 \%)$ & $39(9.2 \%)$ & $74(10.3 \%)$ & 0.53 \\
\hline Dyslipidemia $(N, \%)$ & $604(52.8 \%)$ & $373(51.9 \%)$ & $231(54.2 \%)$ & 0.44 \\
\hline $\mathrm{HbA}_{1 \mathrm{c}}(\%)$ & $8.64 \pm 2.43$ & $8.54 \pm 2.37$ & $8.80 \pm 2.52$ & 0.04 \\
\hline FPG (mmol/L) & $9.25 \pm 3.89$ & $9.24 \pm 3.47$ & $9.26 \pm 4.12$ & 0.92 \\
\hline $2 \mathrm{~h}-\mathrm{PG}(\mathrm{mmol} / \mathrm{L})$ & $17.47 \pm 5.89$ & $17.24 \pm 5.18$ & $17.61 \pm 6.27$ & 0.14 \\
\hline FINS (mU/L) & $14.55 \pm 49.88$ & $14.25 \pm 51.14$ & $15.06 \pm 47.73$ & 0.73 \\
\hline 2h-INS (mU/L) & $32.57 \pm 28.04$ & $31.77 \pm 25.10$ & $33.91 \pm 32.41$ & 0.18 \\
\hline $\mathrm{FCP}(\mathrm{pmol} / \mathrm{L})$ & $651.81 \pm 531.62$ & $649.36 \pm 514.26$ & $655.96 \pm 560.28$ & 0.81 \\
\hline 2h-CP (pmol/L) & $1727.33 \pm 1201.89$ & $1732.11 \pm 1223.63$ & $1792.37 \pm 1165.27$ & 0.19 \\
\hline HOMA-IR & $6.05 \pm 20.38$ & $5.67 \pm 15.48$ & $6.70 \pm 26.70$ & 0.43 \\
\hline
\end{tabular}

T2D: type 2 diabetes; DR: diabetic retinopathy; BMI: body mass index; HR: heart rate; SBP: systolic blood pressure; DBP: diastolic blood pressure; $\mathrm{HbA}_{1 \mathrm{c}}$ : glycosylated hemoglobin; FPG: fasting plasma glucose; FINS: fasting insulin; FCP: fasting C peptide; HOMA-IR: homeostasis model assessment of insulin resistance. The diagnosing criteria of hypertension were SBP $\geq 140 \mathrm{mmHg}$ or DBP $\geq 90 \mathrm{mmHg}$ or current administration of antihypertensive drugs. Smoking and drinking were defined as a personal history of smoking/drinking or current smoking/drinking, regardless of the frequency. Dyslipidemia and hyperuricemia were defined by the reference interval provided by the kit, and subjects currently taking lipid-lowering or hypouricemic drugs were also regarded as dyslipidemia or hyperuricemia patients. HOMA-IR was calculated as follows: HOMA - IR = FPG $\times$ FINS/22.5.

TABLE 2: Thyroid indicators corresponding to different severities of DR.

\begin{tabular}{lcccc}
\hline & Non-DR & Mild NPDR & Moderate NPDR & Severe NPDR or PDR \\
\hline Number & 719 & 325 & 44 & 57 \\
TSH $(\mathrm{mU} / \mathrm{L})$ & $1.92 \pm 0.99$ & $1.99 \pm 1.08$ & $1.99 \pm 1.14$ & $1.83 \pm 0.88$ \\
FT4 $(\mathrm{pmol} / \mathrm{L})$ & $13.58 \pm 1.72$ & $13.72 \pm 1.78$ & $13.57 \pm 1.70$ & $13.57 \pm 1.79$ \\
FT3 $(\mathrm{pmol} / \mathrm{L})$ & $4.10 \pm 0.61$ & $4.11 \pm 0.62$ & $4.06 \pm 0.59$ & $4.10 \pm 0.74$ \\
pTPOAb $(N, \%)$ & $94(13.1 \%)$ & $48(14.8 \%)$ & $5(11.4 \%)$ & $6(10.5 \%)$ \\
pTgAb $(N, \%)^{\mathrm{b}}$ & $132(18.4 \%)$ & $56(17.2 \%)$ & $10(22.7 \%)$ & $19(33.3 \%)^{\mathrm{a}}$ \\
\hline
\end{tabular}

TSH: thyroid-stimulating hormone; FT4: free thyroxine; FT3: free triiodothyronine; pTPOAb: positive thyroid peroxidase antibody; pTgAb: positive thyroglobulin antibody; DR: diabetic retinopathy; NPDR: nonproliferative diabetic retinopathy; PDR: proliferative diabetic retinopathy. Data are expressed as mean \pm SD or number and proportion. The superscript a indicates that the value in the corresponding group is significantly higher than the non-DR group $(p<0.05)$. The superscript $b$ indicates that the variation of the thyroid indicator shows a significantly upward trend.

compared with patients without DR, patients with severe NPDR or PDR had a significantly higher positive rate of $\operatorname{TgAb}(33.3 \%$ vs. $18.4 \%, p<0.05)$.

3.3. The Changing Patterns of DR Prevalence with Thyroid Indicators. Serum TSH, FT4, and FT3 were all divided into tertiles, and the DR prevalence corresponding to different tertiles was calculated; at the same time, DR prevalence was also calculated according to the presentation of positive serum thyroid antibodies. The subgroups with the lowest levels of hormones or negative antibodies were regarded as the reference.

As shown in the (a) and (b) in Figure 2, we found that the prevalence of different stages of DR or overall DR was not significantly higher or lower, with the increase of TSH or FT4. The prevalence of DR corresponding to different 

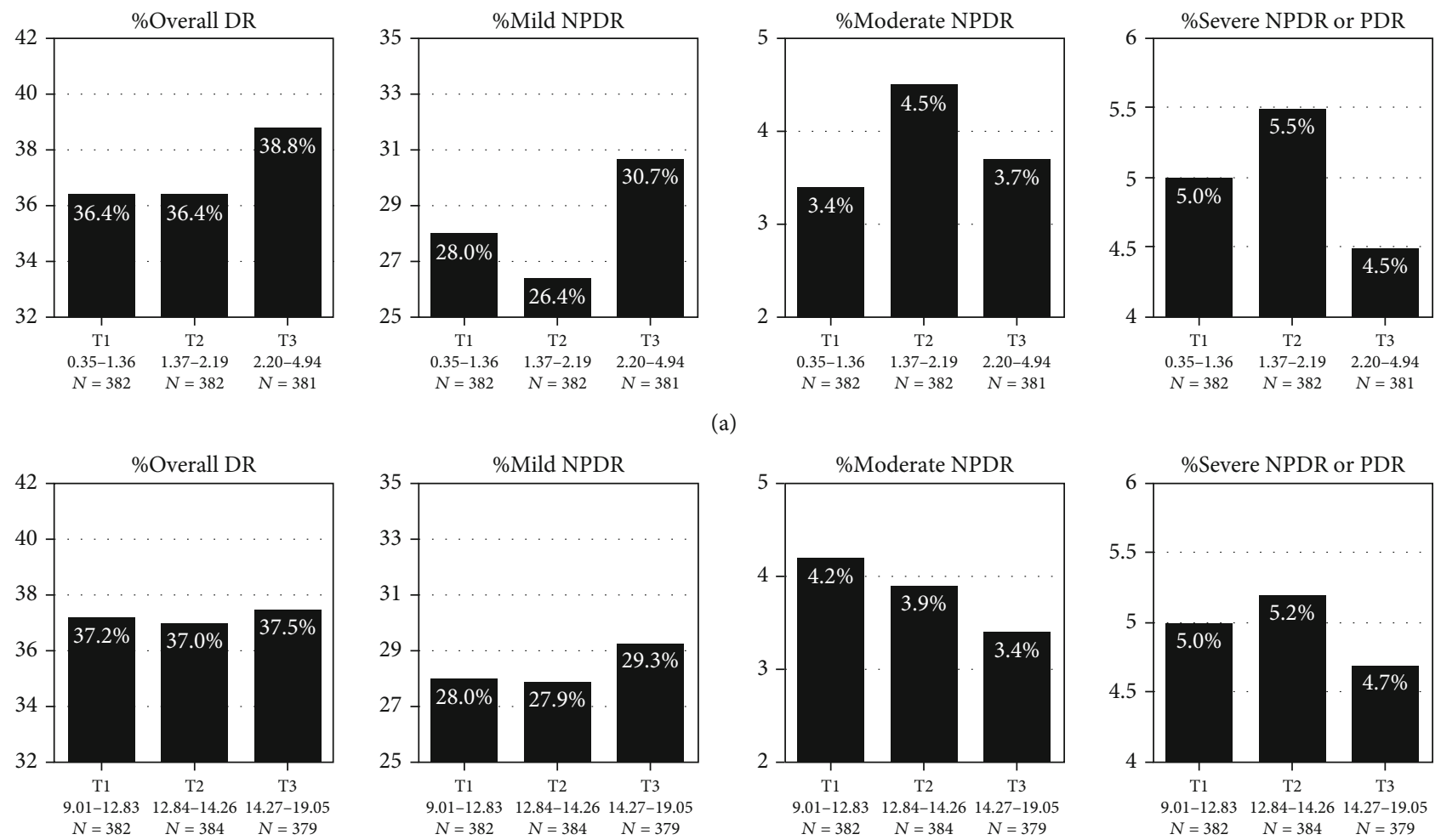

(a)
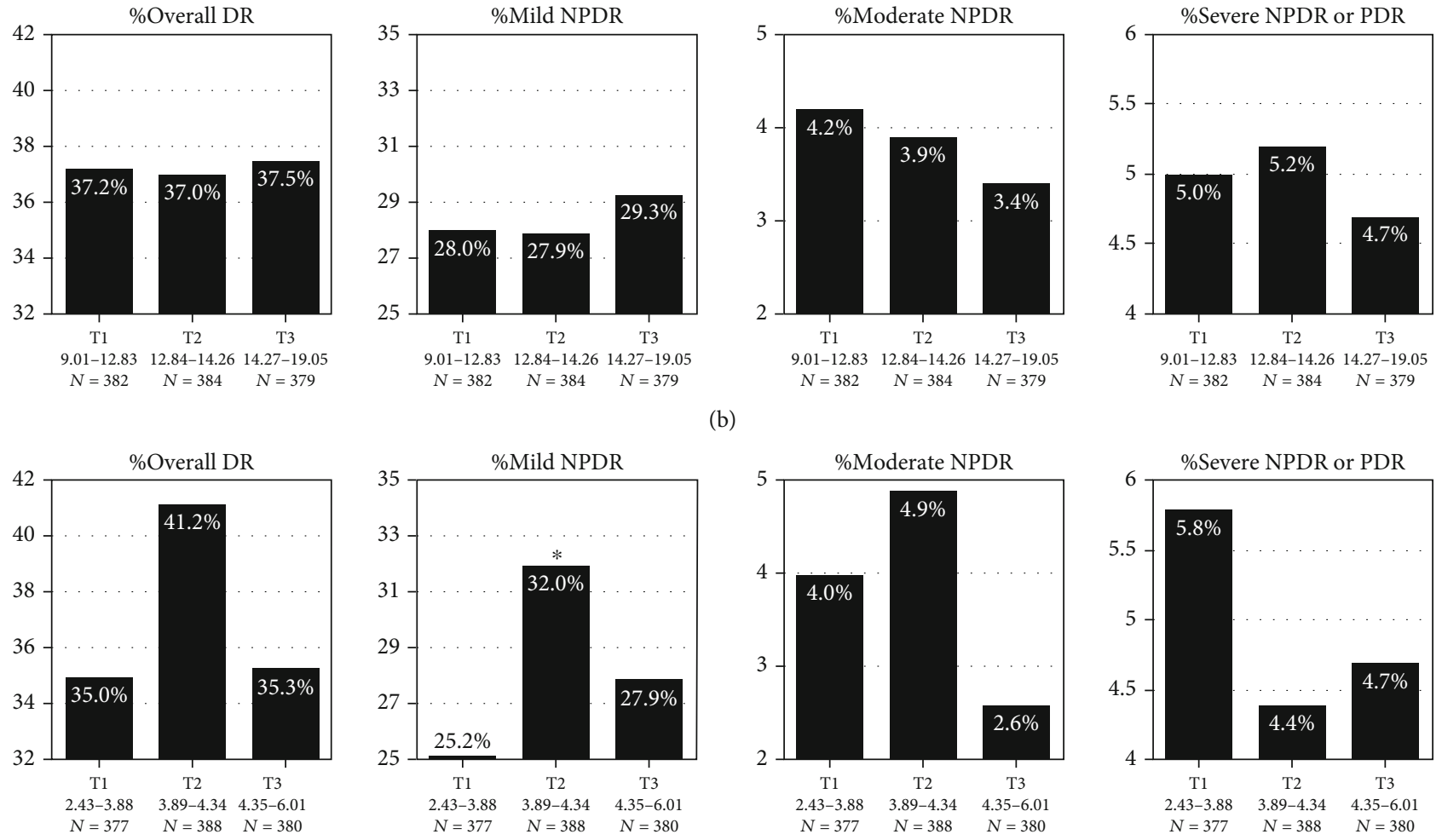

(c)

FIGURE 2: The variation of different stages of DR prevalence with serum TSH (a), FT4 (b), and FT3 (c). DR: diabetic retinopathy; NPDR: nonproliferative diabetic retinopathy; PDR: proliferative diabetic retinopathy; TSH: thyroid-stimulating hormone; FT4: free thyroxine; FT3: free triiodothyronine. The stratification of the thyroid indicators is stated in the figure, and the unit of TSH, FT4, and FT3 should be $\mathrm{mU} / \mathrm{L}, \mathrm{pmol} / \mathrm{L}$, and $\mathrm{pmol} / \mathrm{L}$, respectively. The ${ }^{*}$ indicates that the corresponding prevalence was significantly higher than the control group (T1) $(p<0.05)$.

levels of TSH or FT4 was relatively comparable. In addition, the overall DR prevalence corresponding to different levels of FT3 was also similar. However, the prevalence of mild NPDR in the second tertile (T2) of FT3 was significantly higher $(32.0 \%$ vs. $25.2 \%, p<0.05)$, suggesting that the prevalence of mild NPDR was significantly higher in T2D patients with slightly elevated FT3 within reference interval (3.89-4.34 pmol/L).

Regarding the prevalence of DR under different thyroid autoantibody titers, we found that the DR prevalence did not significantly increase or decrease for T2D patients with positive TPOAb (Figure 3(a)). On the other side, the prevalence of severe NPDR or PDR was significantly higher in TgAb-positive patients ( $8.8 \%$ vs. $4.1 \%, p<0.05)$ (Figure 3(b)), while the prevalence of other stages of DR did not show significancy.

\subsection{Regression Analysis between Thyroid Function and DR} Risks. In order to further confirm the association between the thyroid indicators and different grades of DR risk, univariable and multivariable regression analyses were applied to explore the abovementioned differences in DR prevalence (Table 3). The grouping of thyroid indicators was similar to the above description, and then, the groups with the lowest tertile and the antibody-negative groups were set as the reference. 

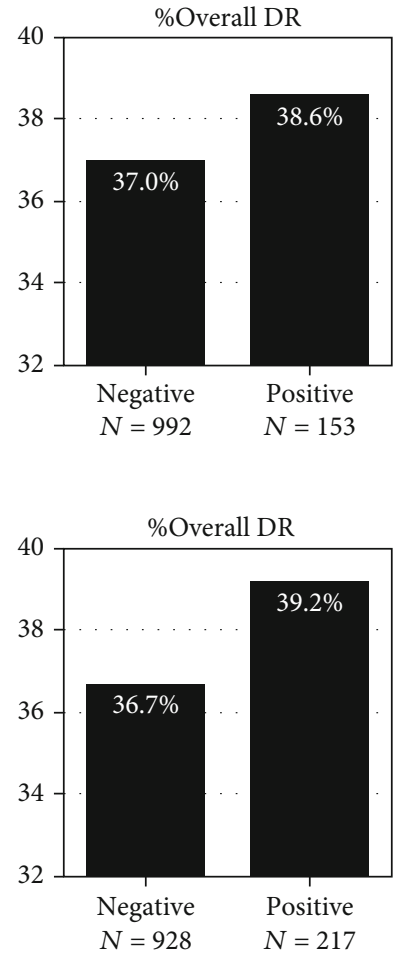
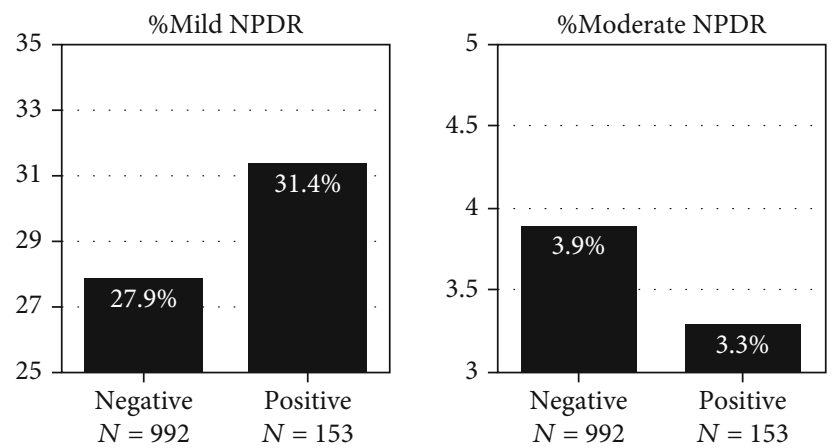

(a)
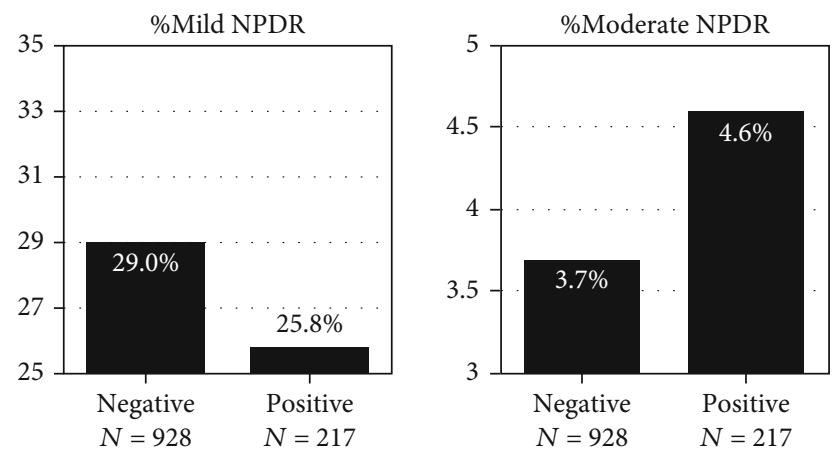
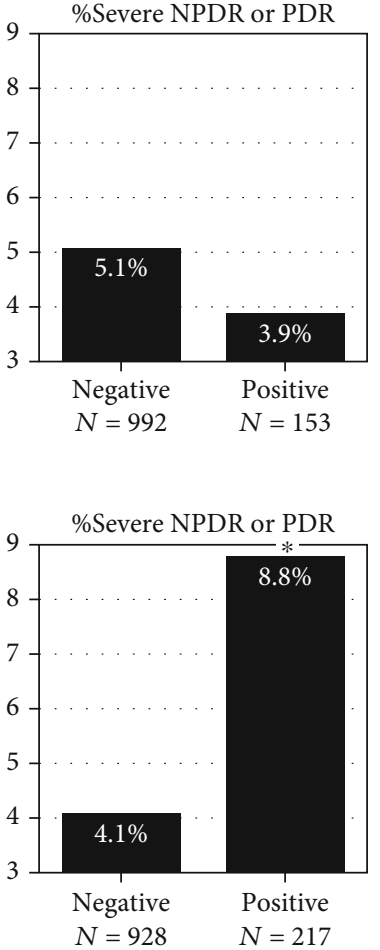

(b)

FIGURE 3: The variation of different stages of DR prevalence with serum TPOAb (a) and TgAb (b). DR: diabetic retinopathy; NPDR: nonproliferative diabetic retinopathy; PDR: proliferative diabetic retinopathy; TPOAb: thyroid peroxidase antibody; TgAb: thyroglobulin antibody. The ${ }^{*}$ indicates that the corresponding prevalence in the positive group was significantly higher than the negative group $(p<0.05)$.

As shown in Table 3, the T2 of FT3 correlated with the risk of mild NPDR (OR $1.426(1.031,1.971), p<0.05)$. What is more, $\mathrm{TgAb}$ positivity was also significantly associated with the risk of severe NPDR or PDR (OR 2.212 (1.244, $3.934), p<0.05)$. The above correlations were both significant in both model 1 and model 2 . However, our results were unable to deduce the significant relationships between the variations in TSH or FT4 within the reference range or the TPOAb positivity and the risk of DR. In addition, we also explored whether the risk of each stage of DR and overall DR would change significantly with the linear changes of TSH, FT4, and FT3. However, with per SD-increase in the abovementioned three indicators, there was no significant variation in the risk of DR.

\section{Discussion}

In this hospital-based retrospective survey, 1,145 T2D patients with normal thyroid function were included. We found that a slight increase in FT3 (3.89-4.34 pmol/L) within the reference range may be significantly associated with the risk of mild NPDR. Patients with a slightly higher FT3 within the specific range had a significantly higher prevalence of mild NPDR. It should be emphasized that although the mild increase in serum FT3 is significantly correlated with mild NPDR, this conclusion does not have much significance in clinical practice. More research is needed to explore the potential association between FT3 levels beyond the reference interval and different stages of DR. In addition, we found that the prevalence of severe NPDR or PDR in $\mathrm{TgAb}$-positive patients was also significantly higher and vice versa. TgAb positivity may also be an independent risk factor for severe DR in euthyroid T2D patients.

The anthropometric and biochemical information were compared in patients with or without DR. We found that compared with non-DR patients, T2D patients with DR are significantly older and have significantly lower levels of $\mathrm{BMI}$, and there are also slight differences in the prevalence of hypertension between the two groups, especially in the significantly higher SBP in DR patients. Compared with patients without DR, patients with DR had a slightly increased prevalence of dyslipidemia and hyperuricemia. In addition, similar to our inherent knowledge that T2D patients with complications generally have worse control of blood glucose than noncomplicated patients, the DR group showed a higher $\mathrm{HbA}_{1 \mathrm{c}}$, and fasting and 2-hour plasma indicators, as well as insulin resistance level also showed insignificant but slight worsen trend.

As mentioned above, several previous studies have been conducted on DR risk and thyroid function, especially $\mathrm{SCH}$, but the conclusion is not clear yet. Similar to the conclusions of several individual studies [24-27], Han et al. have meta-analyzed ten Chinese studies and found that T2D patients with SCH were more likely to be accompanied with DR, and the risk could be increased by $42 \%$ [12]. However, a recent Indian study found that not only DR but other 
TABLE 3: Associations between thyroid indicators and various stages of DR risk.

\begin{tabular}{|c|c|c|c|c|c|}
\hline & & Overall DR & Mild NPDR & Moderate NPDR & Severe NPDR or PDR \\
\hline \multirow{7}{*}{ TSH } & T1 (ref) & 1 & 1 & 1 & 1 \\
\hline & T2 (model 1) & $1.000(0.745,1.343)$ & $0.924(0.672,1.271)$ & $1.322(0.633,2.761)$ & $1.111(0.588,2.102)$ \\
\hline & T2 (model 2) & $0.990(0.734,1.333)$ & $0.917(0.665,1.264)$ & $1.274(0.607,2.674)$ & $1.133(0.596,2.152)$ \\
\hline & T3 (model 1) & $1.110(0.828,1.489)$ & $1.139(0.834,1.556)$ & $1.083(0.502,2.335)$ & $0.892(0.456,1.744)$ \\
\hline & T3 (model 2) & $1.062(0.789,1.431)$ & $1.116(0.814,1.529)$ & $0.954(0.437,2.081)$ & $0.851(0.431,1.680)$ \\
\hline & Per SD increase (model 1) & $1.055(0.938,1.187)$ & $1.081(0.954,1.225)$ & $1.049(0.783,1.404)$ & $0.893(0.679,1.174)$ \\
\hline & Per SD increase (model 2) & $1.043(0.926,1.175)$ & $1.072(0.945,1.216)$ & $1.017(0.757,1.367)$ & $0.891(0.675,1.176)$ \\
\hline \multirow{7}{*}{ FT4 } & T1 (ref) & 1 & 1 & 1 & 1 \\
\hline & $\mathrm{T} 2$ (model 1$)$ & $0.992(0.740,1.330)$ & $0.993(0.724,1.361)$ & $0.930(0.453,1.909)$ & $1.050(0.551,2.000)$ \\
\hline & T2 (model 2) & $1.035(0.768,1.395)$ & $1.030(0.748,1.418)$ & $0.973(0.471,2.010)$ & $1.073(0.562,2.050)$ \\
\hline & T3 (model 1) & $1.013(0.755,1.358)$ & $1.064(0.777,1.458)$ & $0.813(0.385,1.713)$ & $0.953(0.492,1.845)$ \\
\hline & T3 (model 2) & $0.983(0.728,1.328)$ & $1.069(0.776,1.471)$ & $0.808(0.379,1.726)$ & $0.826(0.421,1.620)$ \\
\hline & Per SD increase (model 1) & $1.035(0.967,1.109)$ & $1.047(0.973,1.127)$ & $0.985(0.827,1.172)$ & $0.983(0.843,1.147)$ \\
\hline & Per SD increase (model 2) & $1.028(0.959,1.102)$ & $1.042(0.968,1.122)$ & $0.984(0.826,1.173)$ & $0.968(0.831,1.128)$ \\
\hline \multirow{7}{*}{ FT3 } & $\mathrm{T} 1$ (ref) & 1 & 1 & 1 & 1 \\
\hline & $\mathrm{T} 2$ (model 1) & $1.302(0.972,1.745)$ & $1.394(1.017,1.912)^{\mathrm{c}}$ & $1.243(0.622,2.483)$ & $0.739(0.386,1.416)$ \\
\hline & T2 (model 2) & $1.349(0.997,1.824)$ & $1.426(1.031,1.971)^{\mathrm{c}}$ & $1.146(0.566,2.320)$ & $0.840(0.431,1.637)$ \\
\hline & T3 (model 1) & $1.011(0.750,1.363)$ & $1.148(0.831,1.586)$ & $0.652(0.289,1.471)$ & $0.802(0.423,1.521)$ \\
\hline & T3 (model 2) & $1.031(0.757,1.405)$ & $1.179(0.844,1.647)$ & $0.588(0.254,1.363)$ & $0.846(0.434,1.646)$ \\
\hline & Per SD increase (model 1) & $1.016(0.837,1.235)$ & $1.051(0.853,1.295)$ & $0.876(0.538,1.425)$ & $0.973(0.632,1.499)$ \\
\hline & Per SD increase (model 2) & $1.016(0.829,1.246)$ & $1.061(0.853,1.318)$ & $0.744(0.445,1.244)$ & $1.047(0.673,1.628)$ \\
\hline \multirow{3}{*}{ TPOAb } & Negative & 1 & 1 & 1 & 1 \\
\hline & Positive (model 1) & $1.069(0.753,1.517)$ & $1.180(0.816,1.706)$ & $0.826(0.320,2.128)$ & $0.753(0.318,1.786)$ \\
\hline & Positive (model 2) & $1.085(0.761,1.547)$ & $1.191(0.822,1.726)$ & $0.859(0.332,2.226)$ & $0.749(0.314,1.785)$ \\
\hline \multirow{3}{*}{$\operatorname{TgAb}$} & Negative & 1 & 1 & 1 & 1 \\
\hline & Positive (model 1) & $1.108(0.818,1.502)$ & $0.852(0.609,1.192)$ & $1.270(0.618,2.613)$ & $2.247(1.269,3.981)^{\mathrm{c}}$ \\
\hline & Positive (model 2) & $1.112(0.818,1.511)$ & $0.849(0.606,1.190)$ & $1.343(0.649,2.780)$ & $2.212(1.244,3.934)^{\mathrm{C}}$ \\
\hline
\end{tabular}

Model 1: crude; Model 2: adjusted for age, sex, BMI, SBP, and $\mathrm{HbA}_{1 \mathrm{c}}$. The superscript $\mathrm{c}$ indicates that the $p$ value of corresponding OR is less than 0.05 .

T2D complications such as nephropathy, neuropathy, or cardiovascular disease were not significantly related with any forms of thyroid dysfunction [28]. What is more, several studies also concluded that supranormal or high-normal TSH level was not related with DR, while the risk of nephropathy showed significance [29-31].

Therefore, currently, we have insufficient evidence to support an explicit relationship between thyroid dysfunction (especially $\mathrm{SCH}$ ) and DR. Compared with the above studies, there are fewer studies on thyroid function and DR risk among euthyroid T2D subjects. In a similar hospital-based retrospective study [32], Zou et al. revealed that the prevalence of DR decreased significantly with the increase of serum FT3 level among euthyroid subjects, and the level of FT3 also decreased significantly with the severity of DR. However, the level of TSH or FT4 or the positivity of TPOAb showed no significant trends. Different from the above findings, Kong et al. found that the DR prevalence showed a significantly decreasing trend across the tertiles of FT4, plus a significantly increasing trend across the tertiles of TSH. However, the level of FT3 hardly showed any significance regarding DR or PDR [33]. We speculate that the scales of the previous studies were small, so sampling bias might lead to the inconsistency of the conclusions. In addition, the kits used in various studies are different, so the inconsistent reference intervals for TSH, FT3, or FT4 might also contribute to inconsistent conclusions. Finally, the relevant studies so far are based on hospital-based retrospective populations, so untreated or undiagnosed T2D patients were ignored. It remains to be further explored that whether these evidence truly revealed the association between thyroid indicators and DR risk among euthyroid T2D patients.

Compared with the previous findings, the results of the present research are somewhat different. The prevalence of overall or each stage of DR showed no significant difference across the tertiles of TSH or FT4. However, the prevalence of mild NPDR in the T2 of FT3 was significantly higher than in the T1 of FT3. The following regression analysis further confirmed the above difference in prevalence. We found that when serum FT3 was in the range of T2 (3.89-4.34 mmol/L), the risk of mild NPDR was significantly associated with it. However, when FT3 continues to rise to the level of $\mathrm{T} 3$, the above association has no 
statistical significance. Unlike the previous negative correlation, we consider that the slight increase in FT3 within the reference range may be positively correlated with the risk of mild NPDR. Although previous meta-analysis found that $\mathrm{SCH}$ might be related to the risk of DR, there are currently very few relevant studies carried out in euthyroid T2D patients. Based on the evidence from previous studies, we cannot conclude whether changes in thyroid hormones within the reference range will affect the risk of DR in euthyroid patients with T2D. Our study proposed for the first time a significant association between mildly elevated FT3 and mild DR in euthyroid T2D patients, which is a good supplement to the existing evidence.

Because of the common cooccurrence of autoimmune diseases [34], there have been a number of relevant studies on thyroid autoimmune and DR in T1D patients [35], but we could hardly find any explicit findings on T2D patients. In this study, we found a significant positive correlation between $\operatorname{TgAb}$ positivity and the risk of severe NPDR or PDR among euthyroid T2D patients. This is the first ever explicit conclusion about the relationship between thyroid autoimmunity and T2D-DR. Therefore, more future evidence is required to confirm such a correlation between thyroid autoimmune and the complications of T2D. A variety of inflammatory cytokines in serum have been confirmed to be closely related to the occurrence and development of T2D-DR [36-38]. At the same time, a large number of studies also showed that a variety of circulating cytokines involving in the occurrence and development of thyroid autoimmunity [39-41]. We speculate that the above two groups of pathological processes might overlap or promote each other, which induces a higher $\mathrm{TgAb}$ titer in patients with severe $\mathrm{DR}$ and vice versa. In view of the fact that there are few basic investigations in the related fields at present, more research is needed for in-depth exploration in the future. Although the manifestations of autoimmune thyroid disease (AITD) are generally ignored in practice, it has a certain impact on long-term thyroid function. Given that severe DR patients have a significantly higher TgAb positive rate, it is necessary to discuss in more depth whether it is essential to screen for serum thyroid autoantibodies in T2D patients with severe DR.

This study has some limitations. Firstly, this is a retrospective survey, so we cannot know the causal relationship between thyroid function and DR. Secondly, the current treatment rate of diabetes in mainland China is less than half [2]. Therefore, whether this hospital-based survey reflects the actual association between thyroid function and DR risk in general T2D patients remains unknown.

\section{Conclusions}

In summary, we hardly found that the risks of various stages of DR showed a significant upward or downward trend with serum TSH or thyroid hormone within the reference interval. Although mildly elevated FT3 may be associated with an increased risk of mild NPDR, the extensibility of this finding remains to be explored. In addition, there is a significant positive correlation between $\mathrm{TgAb}$ positivity and severe DR. The positive rate of TgAb in severe NPDR and PDR patients is much higher than that in non-DR T2D patients and vice versa. Further prospective studies are needed to get enough evidence on the association between the thyroid autoantibodies and the presence or development of severe DR.

\section{Data Availability}

The database of the study is available on request to Prof. Lei Liu (email address: liuleijiao@163.com).

\section{Conflicts of Interest}

The authors declare that there is no conflict of interest regarding the publication of this paper.

\section{Authors' Contributions}

Xiaotong Gao and Xichang Wang contributed equally to this work.

\section{Acknowledgments}

This study was funded by the Key Laboratory Project of Thyroid Diseases, National Health Commission (Grant No. 2019PT330001).

\section{References}

[1] Y. Li, D. Teng, J. Ba et al., "Efficacy and safety of long-term universal salt iodization on thyroid disorders: epidemiological evidence from 31 provinces of mainland China," Thyroid, vol. 30, no. 4, pp. 568-579, 2020.

[2] Y. Li, D. Teng, X. Shi et al., "Prevalence of diabetes recorded in mainland China using 2018 diagnostic criteria from the American Diabetes Association: national cross sectional study," BMJ, vol. 369, article m997, 2020.

[3] B. Biondi, G. J. Kahaly, and R. P. Robertson, "Thyroid dysfunction and diabetes mellitus: two closely associated disorders," Endocrine Reviews, vol. 40, no. 3, pp. 789-824, 2019.

[4] W. T. Cefalu, E. G. Berg, M. Saraco, M. P. Petersen, S. Uelmen, and S. Robinson, "2. Classification and diagnosis of diabetes: standards of medical care in diabetes-2019," Diabetes Care, vol. 42, Supplement 1, pp. S13-S28, 2019.

[5] R. H. Chen, H. Y. Chen, K. M. Man et al., "Thyroid diseases increased the risk of type 2 diabetes mellitus: a nation-wide cohort study," Medicine (Baltimore), vol. 98, no. 20, article e15631, 2019.

[6] S. A. Chubb, W. A. Davis, Z. Inman, and T. M. Davis, "Prevalence and progression of subclinical hypothyroidism in women with type 2 diabetes: the Fremantle Diabetes Study," Clinical Endocrinology, vol. 62, no. 4, pp. 480-486, 2005.

[7] M. F. Celani, M. E. Bonati, and N. Stucci, "Prevalence of abnormal thyrotropin concentrations measured by a sensitive assay in patients with type 2 diabetes mellitus," Diabetes Research, vol. 27, no. 1, pp. 15-25, 1994.

[8] P. Perros, R. J. McCrimmon, G. Shaw, and B. M. Frier, "Frequency of thyroid dysfunction in diabetic patients: value of annual screening," Diabetic Medicine, vol. 12, no. 7, pp. 622-627, 1995. 
[9] M. Al-Geffari, N. A. Ahmad, A. H. Al-Sharqawi, A. M. Youssef, D. Alnaqeb, and K. Al-Rubeaan, "Risk factors for thyroid dysfunction among type 2 diabetic patients in a highly diabetes mellitus prevalent society," International Journal of Endocrinology, vol. 2013, Article ID 417920, 2013.

[10] J. J. Diez and P. Iglesias, "An analysis of the relative risk for hypothyroidism in patients with type 2 diabetes," Diabetic Medicine, vol. 29, no. 12, pp. 1510-1514, 2012.

[11] F. Song, C. Bao, M. Deng et al., "The prevalence and determinants of hypothyroidism in hospitalized patients with type 2 diabetes mellitus," Endocrine, vol. 55, no. 1, pp. 179-185, 2017.

[12] C. Han, X. He, X. Xia et al., "Subclinical hypothyroidism and type 2 diabetes: a systematic review and meta-analysis," PLoS One, vol. 10, no. 8, article e0135233, 2015.

[13] Y. Zhu, F. Xu, J. Shen et al., "Prevalence of thyroid dysfunction in older Chinese patients with type 2 diabetes-a multicenter cross-sectional observational study across China," PLoS One, vol. 14, no. 5, article e0216151, 2019.

[14] J. J. Diez and P. Iglesias, "Subclinical hyperthyroidism in patients with type 2 diabetes," Endocrine, vol. 42, no. 1, pp. 157-163, 2012.

[15] T. Zhang, J. Shi, Y. Peng et al., "Sex-influenced association between free triiodothyronine levels and poor glycemic control in euthyroid patients with type 2 diabetes mellitus," Journal of Diabetes and its Complications, vol. 34, no. 11, article 107701, 2020.

[16] X. Zhang, J. Sun, W. Han et al., "The type 2 deiodinase Thr92Ala polymorphism is associated with worse glycemic control in patients with type 2 diabetes mellitus: a systematic review and meta-analysis," Journal Diabetes Research, vol. 2016, article 5928726, 2016.

[17] J. B. Zhou, H. B. Li, X. R. Zhu, H. L. Song, Y. Y. Zhao, and J. K. Yang, "Subclinical hypothyroidism and the risk of chronic kidney disease in T2D subjects: a case-control and dose-response analysis," Medicine (Baltimore), vol. 96, no. 15, article e6519, 2017.

[18] L. Wang, T. Chen, J. Yu, H. Yuan, X. Deng, and Z. Zhao, "Clinical associations of thyroid hormone levels with the risk of atherosclerosis in euthyroid type 2 diabetic patients in Central China," International Journal of Endocrinology, vol. 2020, Article ID 2172781, 2020.

[19] Y. Chen, W. Zhang, N. Wang et al., "Thyroid parameters and kidney disorder in type 2 diabetes: results from the METAL study," Journal Diabetes Research, vol. 2020, article 4798947, 2020.

[20] N. Cheung, P. Mitchell, and T. Y. Wong, "Diabetic retinopathy," Lancet, vol. 376, no. 9735, pp. 124-136, 2010.

[21] M. S. Parmar, "Evaluation and care of patients with diabetic retinopathy," The New England Journal of Medicine, vol. 383, no. 5, article e31, 2020.

[22] L. Yao, Y. Zhong, L. He et al., "Serum CA125 level is associated with diabetic retinopathy in Chinese patients with type 2 diabetes," Diabetes, Metabolic Syndrome and Obesity, vol. 13, pp. 1803-1812, 2020.

[23] ETDRSR Group, "Grading diabetic retinopathy from stereoscopic color fundus photographs-an extension of the modified Airlie House classification," Ophthalmology, vol. 98, no. 5, pp. 786-806, 1991.

[24] J. K. Yang, W. Liu, J. Shi, and Y. B. Li, “An association between subclinical hypothyroidism and sight-threatening diabetic ret- inopathy in type 2 diabetic patients," Diabetes Care, vol. 33, no. 5, pp. 1018-1020, 2010.

[25] G. R. Yang, J. K. Yang, L. Zhang, Y. H. An, and J. K. Lu, “Association between subclinical hypothyroidism and proliferative diabetic retinopathy in type 2 diabetic patients: a casecontrol study," The Tohoku Journal of Experimental Medicine, vol. 222, no. 4, pp. 303-310, 2010.

[26] B. Y. Kim, C. H. Kim, C. H. Jung, J. O. Mok, K. I. Suh, and S. K. Kang, "Association between subclinical hypothyroidism and severe diabetic retinopathy in Korean patients with type 2 diabetes," Endocrine Journal, vol. 58, no. 12, pp. 1065-1070, 2011.

[27] N. Reddy, T. V. S. Pradeep, S. Tirupati, V. Sarathi, and D. Kumar, "Thyroid dysfunction and its association with microvascular complications in patients with type 2 diabetes mellitus in South India," Diabetes and Metabolic Syndrome: Clinical Research and Reviews, vol. 14, no. 4, pp. 615-617, 2020.

[28] V. Mehalingam, J. Sahoo, Z. Bobby, and K. V. Vinod, "Thyroid dysfunction in patients with type 2 diabetes mellitus and its association with diabetic complications," Journal of Family Medicine and Primary Care, vol. 9, no. 8, pp. 4277-4281, 2020.

[29] J. N. Ramis, C. F. Artigas, M. A. Santiago, F. J. Manes, R. S. Canonge, and L. M. Comas, "Is there a relationship between TSH levels and diabetic retinopathy in the Caucasian population?," Diabetes Research and Clinical Practice, vol. 97, no. 3, pp. e45-e47, 2012.

[30] Q. Qi, Q. M. Zhang, C. J. Li et al., “Association of thyroidstimulating hormone levels with microvascular complications in type 2 diabetes patients," Medical Science Monitor, vol. 23, pp. 2715-2720, 2017.

[31] H. S. Chen, T. E. Wu, T. S. Jap et al., "Subclinical hypothyroidism is a risk factor for nephropathy and cardiovascular diseases in type 2 diabetic patients," Diabetic Medicine, vol. 24, no. 12, pp. 1336-1344, 2007.

[32] J. Zou, Z. Li, F. Tian et al., "Association between normal thyroid hormones and diabetic retinopathy in patients with type 2 diabetes," BioMed Research International, vol. 2020, Article ID 8161797, 2020.

[33] X. Kong, J. Wang, G. Gao et al., “Association between free thyroxine levels and diabetic retinopathy in euthyroid patients with type 2 diabetes mellitus," Endocrine Research, vol. 45, no. 2, pp. 111-118, 2020.

[34] V. K. Kawai, M. Shi, Q. Feng et al., "Pleiotropy in the genetic predisposition to rheumatoid arthritis: a phenome-wide association study and inverse variance-weighted meta-analysis," Arthritis \& Rheumatology, vol. 72, no. 9, pp. 1483-1492, 2020.

[35] A. Rogowicz-Frontczak, B. Falkowski, A. Grzelka-Wozniak, A. Uruska, A. Araszkiewicz, and D. Zozulinska-Ziolkiewicz, "Does autoimmune hypothyroidism increase the risk of neurovascular complications in type 1 diabetes?," Journal of Endocrinological Investigation, vol. 43, no. 6, pp. 833-839, 2020.

[36] M. Nalini, B. V. Raghavulu, A. Annapurna, P. Avinash, and V. Chandi, "Correlation of various serum biomarkers with the severity of diabetic retinopathy," Diabetes and Metabolic Syndrome: Clinical Research and Reviews, vol. 11, Supplement 1, pp. S451-S454, 2017.

[37] I. Chatziralli, T. N. Sergentanis, R. Crosby-Nwaobi et al., "Model for risk-based screening of diabetic retinopathy in people with newly-diagnosed type 2 diabetes mellitus," Investigative Ophthalmology \& Visual Science, vol. 58, no. 6, p. BIO99, 2017. 
[38] L. B. Lamine, A. Turki, G. Al-Khateeb et al., "Elevation in circulating soluble CD40 ligand concentrations in type 2 diabetic retinopathy and association with its severity," Experimental and Clinical Endocrinology \& Diabetes, vol. 128, no. 5, pp. 319-324, 2020.

[39] D. Arpaci, S. Karakas Celik, M. Can et al., "Increased serum levels of IL-28 and IL-29 and the protective effect of IL28B rs8099917 polymorphism in patients with Hashimoto's thyroiditis," Immunological Investigations, vol. 45, no. 7 , pp. $668-678,2016$.

[40] L. Sieminska, C. Wojciechowska, B. Kos-Kudla et al., "Serum concentrations of leptin, adiponectin, and interleukin-6 in postmenopausal women with Hashimoto's thyroiditis," Endokrynologia Polska, vol. 61, no. 1, pp. 112-116, 2010.

[41] Q. Guo, Y. Wu, Y. Hou et al., "Cytokine secretion and pyroptosis of thyroid follicular cells mediated by enhanced NLRP3, NLRP1, NLRC4, and AIM2 inflammasomes are associated with autoimmune thyroiditis," Frontiers in Immunology, vol. 9, p. 1197, 2018. 